\title{
Buen vivir y pueblos indígenas en Colombia
}

\author{
Oscar David Montero de la Rosa \\ Indígena del Pueblo Kankuamo, Sierra Nevada de Santa Marta \\ Politólogo Universidad Nacional de Colombia \\ e-mail: oscardavidmontero@gmail.com
}

\begin{abstract}
Resumen
A partir de la Constitución Política de 1991, Colombia se reconoce como un país multiétnico y pluricultural. Los 102 Pueblos Indígenas existentes en el país tienen como principios de lucha el Territorio, la Cultura, la Autonomía y la Unidad. Hoy el movimiento indígena colombiano, es un referente consolidado, a pesar del exterminio físico y cultural. Actualmente 36 pueblos están en riesgo de extinción, a raíz de las políticas estatales, el conflicto armado interno y el desarrollo occidental. El movimiento indígena platea alternativas propias de acuerdo a sus usos y costumbres para una sociedad diversa e intercultural para el Buen Vivir de la humanidad. Este trabajo tiene como objetivo presentar las dinámicas sociales, culturales y políticas de los Pueblos Indígenas, como sujetos políticos colectivos.
\end{abstract}

Palavras-clave: Buen Vivir, territorios indígenas, madre tierra y cosmovisión.

\begin{abstract}
Colombia is a multiethnic and pluri-cultural country according to article 7 of the Political Constitution of Colombia. Achievement that was given by the resistance and cohesion of the indigenous movement, despite being only $3.28 \%$ of the Colombian population, where there are 102 indigenous peoples, which has as principles of struggle: territory, culture, autonomy and unity. Today the Colombian indigenous movement is a reference for the social movements of the country and the Abya Yala movement of peace that raises own alternatives according to their uses and customs for a diverse and intercultural society for the good living of humanity. Despite the fact that extermination in which it lives both physically and culturally, since there are 36 peoples in extinction, as a result of the state policies, the internal armed conflict and the development proposal posed by the West. This research project aims to explain the social, cultural and political dynamics that indigenous peoples have as collective political subjects with respect to the territory and the Good Living that is lived in it. Re meaning the traditions, the uses and imaginaries that we have to maintain our principles of struggle and identity, affected by current problems outside our peoples.
\end{abstract}

Keywords: Good Living, indigenous territories, development, Mother Earth, cosmology, cosmogony, interculturality. 
Introducción

La visión de los Pueblos Indígenas se manifiesta en las formas de relacionarse: Primero, entre los seres humanos, de manera comunitaria, Segundo, con la tierra, como nuestra madre, Tercero, con la naturaleza, pues somos parte integral de ella y no sus dueños.

Rigoberta Menchú Tum ${ }^{1}$ (Discurso de recepción del Premio Nobel de la Paz 1992, citado en Yepes, 2010).

Es el Buen Vivir una propuesta que tienen los pueblos indígenas con sus culturas y sus organizaciones para generar cambios sociales y políticos en el país, a pesar del exterminio físico y cultural en que viven actualmente. Con esta afirmación la investigación busca abordar el tema del Buen Vivir y los Territorios Indígenas en Colombia desde una dimensión integral de las concepciones que tenemos los Pueblos Indígenas y su relación, primero con la Madre Tierra, como ese espacio donde están los padres-madres creadoras y espirituales de todo lo que existe, segundo con la comunidad donde se recrea el pensamiento, la cosmogonía y la universalidad del mundo indígena en lo colectivo, y por último esa relación que se da con los "hermanitos menores"2 (no indígenas), como estrategia de unir y tejer fuerzas para la construcción de un país respetuoso de la diversidad y la diferencia.

Colombia es un país multiétnico y pluricultural, desde la supuesta "conquista", los pueblos originarios del Abya Yala, hemos estado en la lucha y resistencia constante por la reivindicación de nuestros derechos en torno al territorio y la identidad cultural; lo cual se recoge en la propuesta del Buen Vivir, consolidado gracias a los trabajos comunitarios y tradicionales de nuestros pueblos, logrando así en 1991, por medio de la Constitución Política, reconocer a los Pueblos Indígenas como sujetos políticos de acuerdo a sus usos y costumbres, no solo en el ámbito nacional sino también en lo internacional, cuando se logran tratados como el 169 de la OIT-Organización Internacional del Trabajo y la Declaración de las Naciones Unidas sobre los Derechos de los Pueblos Indígenas en el 2007.

En la expresión de una Saga (sabia) del Pueblo Indígena Wiwa de la Sierra Nevada de Santa Marta, para quien el Buen Vivir "[...] no es inventado por la gente de ahora, sino de la gente que había cuando era antes de amanecer (SAGA WIWA, citado en Rocha, 2010, p.28). Lo ratifican los 10 Mandamientos

\footnotetext{
${ }^{1}$ Indígena Guatemalteca, del pueblo Maya -Quiché

${ }^{2}$ Referencia de los indígenas de la Sierra Nevada de Santa Marta, para referirse a la sociedad occidental
} 
para salvar al mundo, planteados por el Gobierno Plurinacional de Bolivia en cabeza de su presidente, el indígena Evo Morales, específicamente el décimo que dice:

Vivir bien, en armonía con la Madre Tierra. No es ninguna novedad el vivir bien, sólo se trata de recuperar la vivencia de nuestros antepasados. No es vivir mejor a costa del otro, es construir una vida comunitaria y en armonía con la Madre Tierra. (Huanacuni, 2010, p. 58).

Es decir como lo plantea Huanacuni (2010).

Vivir bien, es la vida en plenitud. Saber vivir en armonía y equilibrio; en armonía con los ciclos de la Madre Tierra, del cosmos, de la vida y de la historia, y en equilibrio con toda forma de existencia en permanente respeto (p. 32).

Es así, que Buen Vivir, desde los Pueblos Indígenas en Colombia, es estar bien con la Madre Tierra, la comunidad, con todo lo que nos rodea en nuestro territorio, que es nuestra gran casa donde se recrea la vida, la cultura y la identidad de todo indígena; este es un paradigma que se ha venido revitalizando y fortaleciendo dentro de las comunidades, como propuesta alternativa de vida, a lo que plantea hoy la sociedad occidental, con el desarrollo.

Pero es Ecuador, 2008 y Bolivia, 2009, los que en sus constituciones han logrado materializar esta forma de vida, en Ecuador denominado Sumak Kawsay y en Bolivia Sumak Qamaña, en donde dan un cambio estructural a sus países y a la concesión del mundo de percibir la Tierra y todo lo que la integra; y como lo resume claramente Evo Morales, 2009:

Nosotros, los pueblos indígenas del planeta, queremos contribuir a la construcción de un mundo justo, diverso, inclusivo, equilibrado y armónico con la naturaleza para el Vivir Bien de todos los pueblos. Decimos Vivir Bien porque no aspiramos a vivir mejor que los otros. No creemos en la concepción lineal y acumulativa del progreso y el desarrollo ilimitado a costa del otro y de la naturaleza. Tenemos que complementarnos y no competir.

Por último, el Buen Vivir, como forma adecuada de vida, es compartido por otros pensadores de la siguiente manera:

El 'buen vivir' expresa, refiere y concuerda con aquellas demandas de 'decroissance' de Latouche, de 'convivialidad' de Iván Ilich, de 'ecología profunda' de Arnold Naes. El 'buen vivir' 
también recoge las propuestas de descolonización de Aníbal Quijano, Boaventura de Souza Santos, Edgardo Lander, entre otros. El 'buen vivir', es otro de los aportes de los pueblos indígenas del Abya Yala, a los pueblos del mundo, y es parte de su largo camino en la lucha por la descolonización de la vida, de la historia, y del futuro (Davalos, 2008:6, en Quijano, 2012, p. 144).

Con estas concepciones del Buen vivir es pertinente igualmente explicar la relacion y la base donde se fundamenta el Bien Vivir, tales como: las construcciones y significados que hay sobre la Madre tierra, el territorio e igualmente la misma concepción que se tiene del desarrollo.

\section{El Buen Vivir y la importancia para los Pueblos Indígenas}

Cuando se habla de La Madre Tierra esa es para todos los "más de 300 millones de personas que forman alrededor de 5.000 poblaciones indígenas en 70 países del mundo" (Naciones Unidas, 2008), nuestra madre, por tanto debemos cuidarla, protegerla, y entender que es un ser que tiene vida al igual que nosotros. Lo que actualmente el presidente boliviano Evo Morales dice: "la Madre Tierra puede vivir sin el ser humano, pero el ser humano no puede vivir sin la Madre Tierra". Reafirmando que la Madre Tierra es todo para nosotros. (Citado en Huanacuni, 2010, p.35).

La Madre Tierra, es la misma naturaleza, esa que en donde la avaricia de unos, la pone "al servicio del capital" (Acosta y Martínez, 2011, p. 7).

El telón de fondo sobre el que nacen los derechos de la naturaleza, es en gran medida la emergencia planetaria frente a la destrucción de ecosistemas enteros. Emergencia que es el resultado de visiones y prácticas que considera a la naturaleza fuente inagotable de riquezas o depósitos de derechos, "(Acosta y Martínez, 2011, p. 7).

Considerando a la tierra como nuestra madre, y como un ser humano que tiene derechos, se irá logrando y reconociendo su derecho al autogobierno, es así que desde el 2009 se hicieron realidad algunos avances, especialmente en Groenlandia con el logro de tener autonomía sobre sus territorios y en Bolivia con la implementación del 'vivir bien' o 'buen vivir' que recoge los principios de equilibrio y armonía con la naturaleza; complementariedad y reciprocidad en las relaciones sociales y respeto por el medio ambiente. En México, 2003 el EZLN - Ejército Zapatista de Liberación Nacional y el Congreso Nacional Indígena - CNI, iniciaron la puesta en práctica de los Acuerdos a lo largo de sus territorios, creando 
gobiernos indígenas autónomos en Chiapas, Michoacán y Oaxaca, de la misma forma Venezuela, creo el Ministerio del Poder Popular para los Pueblos Indígenas, 2007 y Ecuador se reconoce como un Estado intercultural y plurinacional, dando la categoría de ser humano a la Madre Tierra en la nueva Constitución. Y en la última Conferencia Mundial de los Pueblos sobre el Cambio Climático y los Derechos de la Madre Tierra se decretó que él:

"Reconocimiento de los derechos de la Madre Tierra que comprende los bosques, la selva y todos sus componentes. Para restablecer la armonía con la Madre Tierra, el camino no es ponerle precio a la naturaleza sino reconocer que no sólo los seres humanos tenemos derecho a la vida y a reproducirnos, sino que también la naturaleza tiene derecho a la vida y a regenerarse, y que sin la Madre Tierra los seres humanos no podemos vivir". (Carta de Evo Morales a los pueblos indígenas del mundo, 2010).

Es así como todos los gobiernos y Estados del mundo deben avanzar en este reconocimiento, especialmente Colombia, con el fin de proteger la Madre Tierra de todos los seres que la habitamos. El presidente de Bolivia Evo Morales propone 4 principios mínimos que se deben cumplir, tales como: derecho a la vida, derecho a existir; derecho a la regeneración de su biocapacidad; derecho a una vida limpia, derecho de la Madre Tierra a vivir sin contaminación; y derecho a la armonía y al equilibrio con todos.

Para los Pueblos Indígenas el territorio es lo más importante, dado que sin territorio no hay vida, en él se recrea la identidad, la hermandad y la colectividad. Es en él, donde están los sitios sagrados que dan cuenta del saber milenario de los pueblos. Es así que hay que entender el territorio como un ser integral que tiene vida al igual que todos los seres humanos y que no solo es ese pequeño espacio que pisamos: el territorio lo es todo, es el subsuelo, el aire y todo lo que en la naturaleza hay.

Para los pueblos indígenas el territorio es la base material y espiritual de su existencia. Hay una relación mística con la "Madre Tierra", que es sagrada, es un bien de propiedad colectiva, necesario para la supervivencia del pueblo. Es fuente de vida. Es el arraigo de su historia, cultura, ancestros, autonomía y libertad. Trasciende el concepto occidental de la tierra como accidente físico-geográfico, explotable $y$ comercializable. El territorio indígena es un medio y no un fin. 
(Programa de las Naciones Unidas para el Desarrollo - PNUD, 2012, p. 21).

Los Pueblos Indígenas no somos un obstáculo para el desarrollo, nosotros queremos nuestro propio desarrollo que llamamos Buen Vivir.

El modelo de desarrollo no puede ser capitalista, deben buscarse otros modelos, donde se respeten los ríos, las quebradas, las montañas frágiles; o sea el modelo debe ser uno en donde el ser humano pueda vivir respetando la naturaleza y vivir de ella como parte fundamental de la Tierra. (Sánchez y Molina, 2010, p. 320).

El desarrollo ha significado el deterioro de la naturaleza y la destrucción de nuestras comunidades. "El término desarrollo está ligado a la explotación, a la marginación, a la depredación y a la dependencia, pues desde la lógica occidental, desarrollo implica ganar en desmedro de otro". (Huanacuni, 2010, p. 36).

Por esto el progreso y el desarrollo se convierten así y desde entonces en asuntos de geopolítica global, articulados a formas de dominio imperial/ (neo) colonial, en las que importan los procesos de invisibilizacion y asimilación o reducción, esto como parte de un itinerario de 'salvación'/desarrollo necesario en la expectativa de integración y modernización. (Quijano, 2012, p. 100).

\section{Pueblos Indígenas y la normatividad en Colombia}

El movimiento indígena colombiano está conformado por 102 pueblos, la Organización Nacional Indígena de Colombia (ONIC), es la única organización "que los incluye: los 87 reconocidos por el Estado, 12 reconocidos por las organizaciones indígenas y otros 3 que se auto reconocen desde su condición étnica y cultural", de los cuales "65 hablan su lengua materna" (Ministerio de Cultura, 2009), agrupados en 5 organizaciones de carácter nacional: ONIC, AICO-Autoridades Indígena de Colombia por la Pacha Mama, CIT-Confederación Indígena Tayrona, la OPIAC-Organización de los Pueblos Indígenas de la Amazonia Colombiana y Autoridades Indígenas de Colombia-Gobierno Mayor.

De acuerdo con el censo nacional del Departamento Administrativo Nacional de Estadística (DANE) de 2005, se dice que de los 41.468 .384 de colombianos, hay "1.392.623 indígenas que corresponden al 3,43\% de la población del país". "En la actualidad existen 710 resguardos titulados 
ubicados en 27 departamentos y en 228 municipios del país, que ocupan una extensión de aproximadamente 34 millones de hectáreas, el 29,8\% del territorio nacional". (DANE, 2007, p.19 y 34).

Pero es de mencionar que la ONIC, en su censo interno está reportando más de 3.000.000 millones de indígenas en Colombia.

Los orígenes del movimiento indígena se dan a raíz de los levantamientos y procesos de luchas desde la supuesta conquista, es en este contexto donde el movimiento indígena ve la necesidad de conformar organizaciones de carácter sociopolíticas que represente a la población indígena. Así, en la década de 1960 se da la fundación de la Asociación Nacional de Usuarios Campesinos (ANUC), donde los indígenas hacen parte en la condición de campesinos, pero más tarde se separan por las grandes diferencias que existen entre estos (la más relevante la de la significación de la tierra para estos dos sectores sociales. En el marco del "tercer congreso de la Anuc, en 1974, los indígenas se retiraron" (Sánchez y Molina, 2010, p. 20), con esta separación se logra en 1971 la fundación del Consejo Regional Indígena Cauca (CRIC), el cual años más tarde se da a la tarea de agrupar a todos los indígenas en una organización de carácter nacional, con el fin de enfrentar y frenar las políticas neoliberales del gobierno, dando origen a la ONIC en 1982.

\subsection{Ley Sé3 o Ley de Origen}

Antes del Estado colonial, republicano y ahora Estado Social de Derecho, los Pueblos Indígenas teníamos y tenemos nuestras propias formas de hacer justicia en nuestros territorios ancestrales, de acuerdo a nuestras dinámicas propias, por esto hablar de legislación en Pueblos Indígenas, primero hay que remitirnos a la Ley de Origen, al Derecho Mayor o Ley Natural, es ahí donde están las leyes y normas del indígena, ahí está el principio y el fin de la vida tanto física como espiritual. Esta Ley se materializa en el hacer y el pensar del ser indígena; ella no cambia, se mantiene en el tiempo y en el espacio.

De acuerdo con la plataforma de lucha el movimiento indígena ha logrado incidir en el Estado colombiano de la siguiente manera: en materia constitucional en la Asamblea Nacional Constituyente, de la cual se logró en la Constitución Política de 1991, reconocer que Colombia es un país multiétnico y pluricultural, dejando atrás el modelo homogeneizador que tenía la Constitución de 1886.

\footnotetext{
${ }^{3}$ Palabra en las lenguas indígenas de la Sierra Nevada.
} 


\section{Así, fueron incluidos y reconocidos en el texto de la nueva Constitución los siguientes enunciados:}

Cuadro 1. Derechos indígenas en la Constitución Política de 1991.

\begin{tabular}{|c|c|}
\hline Descripción & Enunciado \\
\hline Artículo:1 & $\begin{array}{l}\text { Colombia es un Estado Social de Derecho [...] con autonomía de sus entidades territoriales, } \\
\text { democrática, participativa y pluralista. }\end{array}$ \\
\hline Artículo: 7 & El Estado reconoce y protege la diversidad étnica y cultural de la Nación. \\
\hline Artículo:10 & $\begin{array}{l}\text { El castellano es el idioma oficial de Colombia. Los idiomas y dialectos de los pueblos } \\
\text { indígenas son también oficiales en sus territorios. La enseñanza que se imparta en las } \\
\text { comunidades con tradiciones lingüísticas propias será bilingüe. } \\
\text { La Ley de Lenguas Nativas: } 1381 / 2010 \text {, reconoce } 68 \text { lenguas: } 65 \text { indígenas, } 2 \text { afro descendientes } \\
\text { (creole y palenquera), y el romaní del pueblo Gitano o RROM. }\end{array}$ \\
\hline Artículo:63 & $\begin{array}{l}\text { Los bienes de uso público, parques naturales, tierras comunales de pueblos indígenas, tierras } \\
\text { de resguardo, el patrimonio arqueológico de la Nación y los demás bienes que determine la } \\
\text { ley, son inalienables, imprescriptibles e inembargables. }\end{array}$ \\
\hline Artículo:68 & $\begin{array}{l}\text { Los integrantes de los pueblos indígenas tendrán derecho a una formación que respete y } \\
\text { desarrolle su identidad cultural }\end{array}$ \\
\hline Artículo:72 & $\begin{array}{l}\text { El patrimonio cultural de la Nación está bajo la protección del Estado. El patrimonio } \\
\text { arqueológico y otros bienes culturales que conforman la identidad nacional, pertenecen a la } \\
\text { Nación y son inalienables, inembargables e imprescriptibles... reglamentarán los derechos } \\
\text { especiales que pudieran tener los pueblos indígenas asentados en territorios de riqueza } \\
\text { arqueológica. }\end{array}$ \\
\hline Artículo:96 & $\begin{array}{l}\text { Son nacionales colombianos: [...] c) Los miembros de los pueblos indígenas que comparten } \\
\text { territorios fronterizos. }\end{array}$ \\
\hline Artículo:171 & $\begin{array}{l}\text { El Senado de la República estará integrado por cien miembros elegidos en circunscripción } \\
\text { nacional. Habrá un número adicional de dos senadores elegidos en circunscripción nacional } \\
\text { especial por comunidades indígenas. }\end{array}$ \\
\hline Artículo:246 & $\begin{array}{l}\text { Las autoridades de los pueblos indígenas podrán ejercer funciones jurisdiccionales dentro de } \\
\text { su ámbito territorial, de conformidad con sus propias normas y procedimientos, siempre que } \\
\text { no sean contrarios a la Constitución y leyes de la República. }\end{array}$ \\
\hline Artículo:286 & $\begin{array}{l}\text { Son entidades territoriales los departamentos, los distritos, los municipios y los territorios } \\
\text { indígenas. }\end{array}$ \\
\hline Artículo:287 & $\begin{array}{l}\text { Las entidades territoriales gozan de autonomía para la gestión de sus intereses, y dentro de } \\
\text { los límites de la Constitución y la ley. En tal virtud tendrán los siguientes derechos: } \\
\text { gobernarse por autoridades propias. }\end{array}$ \\
\hline Artículo:321 & $\begin{array}{l}\text { Las provincias se constituyen con municipios o territorios indígenas circunvecinos, } \\
\text { pertenecientes a un mismo departamento. }\end{array}$ \\
\hline Artículo:329 & $\begin{array}{l}\text { La conformación de las entidades territoriales indígenas se hará con sujeción a lo dispuesto } \\
\text { en la Ley Orgánica de Ordenamiento Territorial, y su delimitación se hará por el Gobierno } \\
\text { Nacional, con participación de los representantes de las comunidades indígenas, previo } \\
\text { concepto de la Comisión de Ordenamiento Territorial. } \\
\text { Los resguardos son de propiedad colectiva y no enajenable. }\end{array}$ \\
\hline Artículo:330 & $\begin{array}{l}\text { De conformidad con la Constitución y las leyes, los territorios indígenas estarán gobernados } \\
\text { por consejos conformados y reglamentados según los usos y costumbres de sus } \\
\text { comunidades... }\end{array}$ \\
\hline Artículo:339 & $\begin{array}{l}\text { [...] Las entidades territoriales elaboraran y adoptaran de manera concertada entre ellas y el } \\
\text { gobierno nacional, planes de desarrollo... }\end{array}$ \\
\hline Artículo:356 & $\begin{array}{l}\text { De la distribución de los Recursos y de las Competencias. } \\
\text { [...] Para efectos serán beneficiarios las entidades territoriales indígenas, una vez } \\
\text { constituidas. Así mismo la, la ley establecerá como beneficiarios a los resguardos indígenas, } \\
\text { siempre y cuando estos no se hayan constituidos en entidad territorial indígena. }\end{array}$ \\
\hline Artículo:360 & $\begin{array}{l}\text { [...] La ley determinara las condiciones para la explotación de los recursos naturales no } \\
\text { renovables así como los derechos de las entidades territoriales sobre los mismos. }\end{array}$ \\
\hline $\begin{array}{l}\text { Artículo } \\
\text { transitorio: } \\
56 .\end{array}$ & $\begin{array}{l}\text { Mientras se expide la ley a que se refiere el artículo } 329 \text {, el gobierno podrá dictar las normas } \\
\text { fiscales necesarias y las demás relativas al funcionamiento de los territorios indígenas y su } \\
\text { coordinación con las demás entidades territoriales. }\end{array}$ \\
\hline
\end{tabular}

Estos desarrollos constitucionales por primera vez en la historia del país hacen visibles la diversidad de los Pueblos Indígenas, situación que tiene 
que leerse y reconocerse como el resultado de las luchas y la resistencia ante la expropiación sistemática de sus territorios.

A partir de la apertura de la Constitución Política de 1991, se logra un pluralismo jurídico positivista en los avances de reconocimiento de la diversidad étnica en Colombia, que permitieron hoy el avance de la expedición de normas interculturales como: Decreto Ley de Víctimas 4633 de 2011, específico para víctimas de los Pueblos Indígenas, en donde se resalta el reconocimiento del territorio como víctima y la condición de víctimas colectivas para los Pueblos Indígenas. El Decreto 1953 de 2014, por el cual se creó un régimen especial para el funcionamiento los territorios indígenas respecto de la administración de los sistemas propios hasta que el congreso expida la ley que trata el artículo 329 de la Constitución Política. Ese decreto reconoce los siguientes Sistemas: Sistema Educativo Indígena Propio- SEIP, Sistema Indígena de Salud Propio e Intercultural- SISPI, Sistema General de Participación- SGP, Planes de Vida, Agua Potable y Saneamiento Básico, Jurisdicción Especial Indígena. El Decreto 2233 de 2014, estableció los mecanismos para la efectiva protección y seguridad jurídica de las tierras y territorios ocupados o poseídos ancestralmente y/o tradicionalmente por los Pueblos Indígenas acorde con lo dispuesto en los artículos 13 y 14 del Convenio 169 de la OIT.

También la Corte Constitucional se ha pronunciado para dar efectividad a las disposiciones de la Constitución. En el marco del Auto 004 de 2009, que define dos importantes directrices para el Estado colombiano. Primero, diseñar e implementar un programa de garantía de derechos de los Pueblos Indígenas afectados por el desplazamiento, y segundo, diseñar e implementar distintos planes de salvaguarda étnica ante el conflicto y desplazamiento forzado para cada uno de los 34 Pueblos Indígenas reconocidos en el Auto, como pueblos en particular riesgo de extinción física y cultural. Dos años después se sumaron dos planes de salvaguarda adicionales para un total de 36 .

En otro punto, tenemos como referencia importante la normatividad internacional adoptada en Colombia por la Ley 21 de 1991, que incorpora el Convenio 169 de la Organización Internacional del Trabajo sobre Pueblos Indígenas y Tribales del mundo. En el mismo sentido, integra las bases normativas de la Declaración de las Naciones Unidas sobre los derechos de los Pueblos Indígenas que enfatizó en su artículo 1, que los indígenas tienen derecho, como pueblos o como individuos, al disfrute pleno de todos los derechos humanos y las libertades fundamentales reconocidas en la Carta de las Naciones Unidas, la Declaración Universal de Derechos Humanos y las normas internacionales de derechos humanos. 


\subsection{Retos del Buen Vivir}

Los Pueblos Indígenas de Colombia, del Abya Yala y del mundo afrontamos una crisis que afecta nuestra identidad, nuestra forma de pensar y de vivir en nuestras comunidades de origen. La globalización, los megaproyectos, la contaminación ambiental, las políticas extractivistas y "desarrollista" del Estado, la presencia de grupos armados en nuestros territorios, la educación y la religión impuestas, y el calentamiento global están ocasionando un constante debilitamiento en el pensamiento milenario y en el buen vivir de los pueblos originarios hijos de la Madre Tierra.

Los principales obstáculos para el Buen Vivir en Colombia son: el conflicto armado-desplazamiento forzado; la legislación colombiana y las políticas capitalistas del Estado. Los Pueblos Indígenas de Colombia, cada día luchan y resisten ante la violencia, el desplazamiento y ante el desarraigo cultural de sus territorios ancestrales al cual no están prestos a renunciar. Sin embargo, existen

34 pueblos en riesgo de desaparición que hacen parte de un grupo de 66 en estado de vulnerabilidad por su baja población y los efectos de otro complejo de "vulnerabilidad" como el desplazamiento forzado, el narcotráfico, la colonización, los megaproyectos, las explotaciones de hidrocarburos, la minería y la extracción de madera (PNUD. 2012).

Diversas organizaciones coinciden en el carácter incremental del desplazamiento forzado indígena en Colombia. El Centro de Cooperación al Indígena (CECOIN), reporta un incremento del desplazamiento forzado indígena: "de más de 5100 personas en el 2002, 4602 en el 2003, 7901 en el 2004, 23700 en 2005, 5487 en 2006; principalmente en los departamentos del Chocó, Guajira, Nariño, Cauca y Huila" (Alvarado, 2006).

El conflicto armado interno no es de los Pueblos Indígenas, ante el cual nos hemos declarado, de manera repetida, autónomos y neutrales, exigiéndole a los grupos armados ilegales y legales que respecten nuestras vidas, nuestra integridad colectiva y nuestros territorios. Para nosotros el territorio es la vida misma.

Este conflicto ajeno a nuestras formas de vida se ha materializado en señalamientos y asesinatos selectivos de líderes y autoridades tradicionales, incursiones, presencia y confrontaciones de grupos armados, ocupación de sitios sagrados, instalación de bases militares y batallones, instalación de minas antipersona. Ese conflicto armado alcanza a las 
comunidades indígenas que además tienen sus territorios impactados por el avance de cultivos de usos ilícitos.

Para superar los impactos del conflicto el movimiento indígena, está implementando la guardia indígena, universidades propias (Universidad Autónoma Indígena Intercultural-UAIIN, Misak Universidad, entre otras), EPS-Indígenas y la Minga como una estrategia de movilización de la palabra de los pueblos, por la vida, la justicia y la autonomía de los territorios, para mantener la identidad como pueblos ancestrales y para seguir recreando la cultura propia del movimiento indígena como un ser colectivo.

Así también sigue otras cuestiones sociales, como la salud, que para los Pueblos Indígenas se relaciona con el vivir bien y el estar bien en la colectividad, de acuerdo con la cosmogonía, es decir, estar bien con la Madre Tierra, con la comunidad, con el cuerpo, tanto en lo físico como en lo mental y espiritual, con la familia y con todo lo que lo rodea. La salud es trasversal a todos los ciclos y momentos de la vida; y se expresa en la relación con el ambiente, las relaciones en la comunidad, la producciónalimentación, las relaciones con otros pueblos y con el Estado. La salud está ligada con el territorio como principal generador de vida, armonía y equilibrio con el cosmos.

El Buen Vivir implica entendimiento de manera integral, que está directamente relacionado con la vida misma y emancipación de los Pueblos Indígenas, de allí su relación con la educación. El movimiento indígena ha luchado por la implementación del Sistema Educativo Indígena Propio SEIP, que busca generar propuestas propias desde las comunidades, logrando así descolonizar el pensamiento y las estructuras educativas de occidente que están en los territorios indígenas.

Este sistema plantea la educación desde una visión integral que abarca todas las etapas vitales del ser humano, es decir este siempre está en constante aprendizaje, que recibe por medio de la palabra y la tradición oral el conocimiento ancestral en los ríos, montañas, sitios sagrados y todo el entorno comunitario en el que se encuentra.

Hay muchas maneras de expresar la resistencia desde el movimiento indígena, y una de sus mejores expresiones es la Guardia Indígena, que es un proceso de resistencia civil y pervivencia en nuestro territorio, en defensa de la vida y de la autonomía de los pueblos indígenas, enmarcados en el Plan de Vida y en respuesta a todos los factores de violencia que atentan contra el bienestar y la armonía de los niños, los jóvenes, los adultos 
y mayores: Basados en la Ley de Origen, el ejercicio del derecho propio y la Constitución Nacional Art. 7, 330 y 246. (NASA-ACIN) (Olsen. 2008, p.2).

Es una de las formas más autóctonas de resistencia civil indígena en el país, originada en el departamento del Cauca con los indígenas Nasas, hoy ha sido tomada como ejemplo casi en todos los Pueblos Indígenas de Colombia, de acuerdo a sus usos y costumbres; no es una fuerza de choque, sino de control, donde su estructura sociopolítica es dependiente e integrada por indígenas de las comunidades, respaldados por las Autoridades Tradicionales y la comunidad; tienen como principal función defender la autonomía territorial y su principio de lucha es la no violencia, su única arma es el pensamiento y el bastón de mando.

En ese escenario de diferentes procesos, igualmente la cultura política se materializa en las percepciones, imaginarios, valores y preocupaciones que tiene el movimiento indígena como sujeto político colectivo. Las decisiones y acciones resultan de esa conciencia.

Por eso una de las estrategias del movimiento indígena, aparte de las movilizaciones y de las Mingas sociales, comunitarias y de resistencias, se han dado paralelamente en el ámbito político, donde se crean dos partidos políticos indígenas en sus inicios la ASI: Alianza Social Indígena, hoy Alianza Social Independiente y AICO- Político, de los cuales por jurisdicción especial se tiene derecho a 2 senadores y un representante a la cámara; fruto de estos partidos se logra en el año 2000 tener el primer gobernador indígena en Colombia y en Latinoamérica, el Taita Floro Tunubala, indígena Misak, como gobernador del departamento del Cauca; hoy la ONIC tiene como fuerza política en lo electoral al MAIS: Movimiento Alternativo Indígena y Social.

Pero está claro que han sido las movilizaciones las que han ayudado a materializar el buen Vivir, y han fortalecido otras propuestas como las resistencias en los territorios, las mismas estructuras políticas organizativas con las organizaciones indígenas, la Minga como espacio y escenario de movilizar la palabra y siempre de la mano las vías Jurídicas para demandar las vulneraciones en lo nacional e internacional.

Finalmente el Buen Vivir se materializa con los Planes de Vida, los cuales son un proceso colectivo donde se plantea y planea todo lo que se quiere para la vida, no son políticas públicas dado que no se hacen para ciertos periodos de gobierno o periodos de tiempo, como en occidente, son políticas de vida de las comunidades, pueblos y organizaciones. 


\section{Consideraciones finales}

Para los Pueblos Indígenas de Colombia, el Buen Vivir y Vivir Bien se materializa en los planes de vida; los sistemas de salud, justicia y educación. Todas las acciones de buen vivir que mantenemos pueden sintetizarse en el cuidado, protección y defensa del territorio, fortalecimiento de los procesos políticos organizativos, la defensa de lo público ante lo cual planteamos sistemas para la vida como el SEIP en educación y el SISPI en Salud, la misma guardia indígena como fuerza común y de base para el control territorial de y para las comunidades.

El Buen Vivir, de nuestros pueblos es mantener el equilibrio con la madre tierra, cuidar y preservar todo lo que en ella hay para logra una vida tranquila y plena, no solo para nosotros, sino para toda la humanidad, ante lo cual invitamos a todos y todas a sumarse a la defensa de la vida y de la madre tierra; porque todos somos sus hijos y nos sus dueños.

\section{Referências Bibliográficas}

ACOSTA A Y MARTÍNEZ E. (Comp.) (2011). La Naturaleza con Derechos: De la Filosofía a la Política. (1era Ed.). Quito. Ediciones Abya-Yala.

COLOMBIA. Congreso de la Republica. Constitución Política de Colombia de 1991.

COLOMBIA. Congreso de la República. Corte Constitucional, AUTO N 004 de 2009, Bogotá, D.C. (2009). Disponible en http://www.corteconstitucional.gov.co/relatoria/autos/2009/a004-09.htm

COLOMBIA. Congreso de la República. Corte Constitucional, AUTO 092 de 2008: Adopción de medidas para la protección a mujeres víctimas del desplazamiento forzado por causa del conflicto armado. Disponible en: http://www.corteconstitucional.gov.co/relatoria/autos/2008/a092-08.htm

COLOMBIA. Congreso de la República. Corte Constitucional, 251 de 2008 : Protección de los derechos fundamentales de los niños, niñas y adolescentes desplazados por el conflicto armado. Disponible: http://www.dnp.gov.co/LinkClick.aspx?fileticket=19i_J5Yh18Y\%3D\&tabid= $\underline{1080}$

COLOMBIA. Ministerio de Cultura: 21 de febrero, Día Mundial de la Lengua Materna y Día Nacional de las Lenguas Nativas. Recuperado en http://www.mincultura.gov.co/?idcategoria=42641 
COLOMBIA. Sistema Educativo Indígena Propio - SEIP. (2011). Operativización del sistema educativo indígena propio del Consejo Regional Indígena del Cauca- CRIC. Popayán

COLOMBIA. Tejido de Comunicación Asociación de Cabildos Indígenas del Norte del Cauca ACIN- CHXAB WALA KIWE, (Productor). (2006). Somos Alzados en Bastones de Mando (documental).

DANE: Departamento Administrativo Nacional de Estadística, dirección de censos y demografías (2007). Colombia una Nación Multicultural: Su Diversidad Étnica. Recuperado en: http://www.dane.gov.co/files/ censo2005/etnia/sys/colombia_nacion.pdf

HUANACUNI F. (2010). Buen Vivir/ Vivir Bien: Filosofías, políticas, estrategias y experiencias regionales andinas. Lima, Perú: ( $1^{\text {a }}$ ed.). Recuperado en http://www.coordinadoracaoi.org/portal/sites/default/ files/Libro\%20\%20Buen\%20Vivir\%20y\%20Vivir\%20Bien.pdf

MINGORANCE F. (2008). Tierra profanada impactos de los megaproyectos en territorios indígenas de Colombia, Cultivos ilícitos megaproyectos. Bogotá, Colombia: ( $1^{\text {a }}$ ed.) Recuperado en: http://cms.onic.org.co/wpcontent/uploads/downloads/2012/03/cartilla_derechospueblos.pdf

NACIONES UNIDAS. (2008). Declaración de las Naciones Unidas sobre los derechos de los pueblos indígenas. Recuperado en: http://www.un.org/esa/socdev/unpfii/documents/DRIPS_es.pdf

NACIONES UNIDAS. Centro de Información: México, Cuba y República Dominicana. Poblaciones indígenas (2008). Recuperado en http://www.cinu.org.mx/temas/p_ind.htm

NACIONES UNIDAS. Programa de las Naciones Unidas para el Desarrollo PNUD, (2012). Pueblos indígenas: diálogo entre culturas, colección Cuadernos INDH 2011. Bogotá, Colombia: Unión Grafica. Recuperado en http://pnud.org.co/img_upload/61626461626434343535373737353535/2012/ cuaderno_indigenas.pdf

OLSEN. V (2008). Marco Legal para los Derechos de los Pueblos indígenas en Colombia. Disponible en: http://cms.onic.org.co/wp-content/uploads/ downloads/2012/03/cartilla_derechospueblos.pdf

ORGANIZACIÓN NACIONAL INDÍGENA DE COLOMBIA ONIC. (2010). "Palabra Dulce, Aire de Vida": Forjando Caminos para la Pervivencia de los Pueblos Indígenas en Riesgo de Extinción en Colombia. Bogotá. Recuperado en Disponible en http://cms.onic.org.co/wp-content/uploads/downloads/ 2012/03/cartilla_palabradulce.pdf 
QUIJANO O. (2012). Ecosimías: Visiones y prácticas de diferencia económico /cultural en contextos de multiplicidad. En Q. Quijano O. (Ed.), Desarrollo, economía y cultura. Entronques y tensiones con formas de dominio imperial/colonial y de geopolítica global. (pp. 91-145). Popayán, Colombia: Editorial Universidad del Cauca/Universidad Andina Simón Bolívar.

ROCHA M. (2010). Antes el amanecer: Antología de las literaturas indígenas de los Andes y la Sierra Nevada de Santa Marta. Bogotá, Colombia: Nomos Impresores ( $1^{\text {a }}$ ed.).

SÁNCHEZ GUTIÉRREZ, E. y MOLINA ECHEVERRI, H. (2010). Documentos para la historia del movimiento indígena colombiano contemporáneo. Bogotá, Colombia: Nomos Impresores ( $1^{\mathrm{a}}$ ed.).

YEPES E. (2010). Los Indígenas Latinoamericanos Hoy. Recuperado de http://www.filosofiadigital.com/2010/09/los-indigenas-latinoamericanoshoy-por-enrique-yepes/ 